\title{
A Novel Magnetic Update Operator for Quantum Evolutionary Algorithms
}

\author{
M. H. Tayarani, N., A. Prugel Bennett and H. Mohammadi
}

\begin{abstract}
Quantum Evolutionary Algorithms (QEA) are novel algorithms proposed for class of combinatorial optimization problems. The probabilistic representation of possible solutions in QEA helps the q-individuals to represent all the search space simultaneously. In QEA, Q-Gate plays the role of update operator and moves qindividuals toward better parts of search space to represent better possible solutions with higher probability. This paper proposes an alternative magnetic update operator for QEA. In the proposed update operator the q-individuals are some magnetic particles attracting each other. The force two particles apply to each other depends on their fitness and their distance. The population has a cellular structure and each q-individual has four neighbors. Each q-individual is attracted by its four binary solution neighbors. The proposed algorithm is tested on Knapsack Problems, Trap problem and fourteen numerical function optimization problems. Experimental results show better performance for the proposed update operator than Q-Gate.
\end{abstract}

\section{Introduction}

Quantum Evolutionary Algorithms are new optimization algorithms proposed for class of combinatorial optimization problems [1]. QEA uses probabilistic representation for possible solutions and this characteristic helps the q-individuals to represent all the search space simultaneously. Several works try to improve the performance of QEA. Combining the concepts of Immune systems and QEA, [2] proposes an immune quantum evolutionary algorithm. In another work [3] proposes a novel particle swarm quantum evolutionary algorithm. A new adaptive rotation gate is proposed in [4] which uses the probability amplitude ratio of the corresponding states of

M. H. Tayarani, N.

University of Southampton, e-mail: mhtn1g09@ece.soton.ac.uk

Adam Prugel Bennett

University of Southampton, e-mail: apb@ecs.soton.ac.uk 
quantum bits. Inspired by the idea of hybrid optimization algorithms, [5] proposes two hybrid-QEA based on combining QEA with PSO. In [6] a novel Multi-universe Parallel Immune QEA is proposed. In the algorithm all the q-individuals are divided into some independent sub-colonies, called universes. Since QEA is proposed for the class of combinatorial optimization problems, [7] proposes a new version of QEA for numerical function optimization problems. A novel quantum coding mechanism for QEA is proposed in [8] to solve the travelling salesman problem. In another work [9] points out some weaknesses of QEA and explains how hitching phenomena can slow down the discovery of optimal solutions. In this algorithm, the attractors moving the population through the search space are replaced at every generation. A new approach based on Evolution Strategies is proposed in [10] to evolve quantum unitary operators which represents the computational algorithm a quantum computer would perform to solve an arbitrary problem. In order to preserve the diversity in population and empower the search ability of QEA, [11] proposes a novel diversity preservation operator for QEA. Reference [12] proposes a sinusoid sized population QEA that makes a tradeoff between exploration and exploitation. While QEA is suitable for combinatorial problems and is relatively weak for real coded problems like numerical function optimization problems, several works have focused on this foible. Reference [13] proposes a probabilistic optimization algorithm, which similar to QEA uses a probabilistic representation for possible solutions.

In QEA, Q-Gate plays the role of update operator and moves the q-individuals toward better parts of the search space. Each q-individual moves toward its best observed possible solution and the only interaction among the q-individuals is the simple copying of best observed binary solutions (see local and global migrations in [1]). This paper proposes a more complicated update operator for QEA, inspiring magnetic field theory which offers more interaction among q-individuals and binary solutions to help the q-individuals extract more information from each other. In the proposed algorithm, the binary solutions attract q-individuals and the binary solutions with higher fitness apply more force to the q-individuals. The proposed algorithm has a parameter and this paper tries to investigate the effect of the parameter on the performance of the proposed algorithm. The proposed algorithm is tested on several benchmark functions including Knapsack problem, Trap problem and numerical function optimization problems. Experimental results show better performance for the proposed update operator than Q-Gate.

This paper is organized as follows. Section 2 introduces Quantum Evolutionary Algorithm and its representation. In section 3 the proposed algorithm is proposed and its parameter is investigated. Experimental results are performed in section 4 and finally section 5 concludes the paper.

\section{QEA}

QEA is inspired from the principles of quantum computation, and its superposition of states is based on qubits, the smallest unit of information stored in a two-state 
quantum computer. A qubit could be either in state "0" or "1", or in any superposition of the two as described below:

$$
|\psi\rangle=\alpha|0\rangle+\beta|1\rangle
$$

Where $\alpha$ and $\beta$ are complex number, which denote the corresponding state appearance probability, following below constraint:

$$
|\alpha|^{2}+|\beta|^{2}=1
$$

This probabilistic representation implies that if there is a system of $m$ qubits, the system can represent $2^{m}$ states simultaneously. At each observation, a qubits quantum state collapses to a single state as determined by its corresponding probabilities.

Consider $i-t h$ individual in $t-t h$ generation defined as an $\mathrm{m}$-qubit as below:

$$
\left[\begin{array}{llllll}
\alpha_{i 1}^{t} & \alpha_{i 2}^{t} & \ldots & \alpha_{i j}^{t} & \ldots & \alpha_{i m}^{t} \\
\beta_{i 1}^{t} & \beta_{i 2}^{t} & \ldots & \beta_{i j}^{t} & \ldots & \beta_{i m}^{t}
\end{array}\right]
$$

Where $\left|\alpha_{i j}^{t}\right|^{2}+\left|\beta_{i j}^{t}\right|^{2}=1, j=1,2, m, m$ is the number of qubits, i.e., the string length of the qubit individual, $i=1,2, n, n$ is the number of possible solution in population and $t$ is generation number of the evolution.

\subsection{QEA Structure}

In the initialization step of QEA, $\left[\begin{array}{ll}\alpha_{i j}^{t} & \beta_{i j}^{t}\end{array}\right]^{T}$ of all $q_{i}^{0}$ are initialized with $\frac{1}{\sqrt{2}}$. This implies that each qubit individual $q_{i}^{0}$ represents the linear superposition of all possible states with equal probability. The next step makes a set of binary instants; $x_{i}^{t}$ by observing $Q(t)=\left\{q_{1}^{t}, q_{2}^{t}, \ldots, q_{n}^{t}\right\}$ states, where $X(t)=/ x_{1}^{t}, x_{2}^{t}, \ldots, x_{n}^{t} /$ at generation $t$ is a random instant of qubit population. Each binary instant, $x_{i}^{t}$ of length $m$, is formed by selecting each bit using the probability of qubit, either $\left|\alpha_{i j}^{t}\right|$ or $\left|\beta_{i j}^{t}\right|$ of $q_{i}^{t}$. Each instant $x_{i}^{t}$ is evaluated to give some measure of its fitness. The initial best solution $b=\max _{i=1}^{n}\left\{f\left(x_{i}^{t}\right)\right\}$ is then selected and stored from among the binary instants of $X(t)$. Then, in 'update' $Q(t)$, quantum gates $U$ update this set of qubit individuals $Q(t)$ as discussed below. This process is repeated in a while loop until convergence is achieved. The appropriate quantum gate is usually designed in accordance with problems under consideration.

\subsection{Quantum Gates Assignment}

The common mutation is a random disturbance of each individual, promoting exploration while also slowing convergence. Here, the quantum bit representation can 
be simply interpreted as a biased mutation operator. Therefore, the current best individual can be used to steer the direction of this mutation operator, which will speed up the convergence. The evolutionary process of quantum individual is completed through the step of "update $Q(t)$ ". A crossover operator, quantum rotation gate, is described below. Specifically, a qubit individual $q_{i}^{t}$ is updated by using the rotation gate $U(\theta)$ in this algorithm. The $j-t h$ qubit value of $i-t h$ quantum individual in generation $t,\left[\begin{array}{ll}\alpha_{i j}^{t} & \beta_{i j}^{t}\end{array}\right]^{T}$ is updated as:

$$
\left[\begin{array}{l}
\alpha_{i j}^{t} \\
\beta_{i j}^{t}
\end{array}\right]=\left[\begin{array}{cc}
\cos (\Delta \theta) & -\sin (\Delta \theta) \\
\sin (\Delta \theta) & \cos (\Delta \theta)
\end{array}\right]\left[\begin{array}{l}
\alpha_{i j}^{t-1} \\
\beta_{i j}^{t-1}
\end{array}\right]
$$

Where $\Delta \theta$ is rotation angle and controls the speed of convergence and determined from Table 1. Reference [14] shows that these values for $\Delta \theta$ have better performance.

Table 1 Lookup Table of $\Delta \theta$, the rotation gate. $x_{i}$ is the $i-t h$ bit of the observed binary solution and $b_{i}$ is the $i-t h$ bit of the best found binary solution.

\begin{tabular}{llll}
\hline$x_{i}$ & $b_{i}$ & $f(x) \geq f(b)$ & $\Delta \theta$ \\
\hline 0 & 0 & false & 0 \\
0 & 0 & true & 0 \\
0 & 1 & false & $0.01 \pi$ \\
0 & 1 & true & 0 \\
1 & 0 & false & $-0.01 \pi$ \\
1 & 0 & true & 0 \\
1 & 1 & false & 0 \\
1 & 1 & true & 0 \\
\hline
\end{tabular}

\section{Magnetic Update Operator}

As it is seen in previous section, Q-Gate has the role of update operator in QEA and moves the q-individuals toward better parts of the search space to represent better possible solutions with higher probability. In each iteration, Q-Gate moves the q-individuals to their best observed possible solutions with the certain value of $\Delta \theta_{i}$. This kind of update operator has two weaknesses. First regardless of the fitness of the best observed possible solution, the q-individuals are moved with a constant value of $\Delta \theta_{i}$, which is the same for various best observed possible solutions with various values of fitness. Second, each q-individual is affected with only one possible solution and other possible solutions have not any effect on the q-individual. In Q-Gate update operator the only interaction among q-individuals is the local and global migrations which are a simple copying of binary solutions. This paper proposes a novel update operator for QEA inspiring from the attraction among magnetic 
particles. Recently we proposed a novel optimization algorithm called Magnetic Optimization Algorithm [14]. In MOA the possible solutions are some magnetic particles attracting each other. Each magnetic particle applies a force to its neighbors, and the amplitude of force is determined by the fitness of the particles and the distance the particles have. Here we propose a similar update operator for QEA. In the proposed update operator, the q-individuals are attracted toward all of their binary solution neighbors. In the proposed update operator even the inferior binary solutions attract the q-individuals and have effect on searching process. The pseudo code of the proposed update operator is as follows:

Procedure Basic MQEA

begin

$t=0$

1. initialize $Q^{0}$

2. while not termination condition do

begin

$t=t+1$

3. make $X^{t}$ by observing the states of $Q^{t-1}$

4. evaluate the particles in $X^{t}$ and store their performance in magnetic fields $B^{t}$

5. normalize $B^{t}$ according to 6

6. evaluate the mass $M^{t}$ for all particles according to 7

7. for all q-individuals $q_{i j}^{t}$ in $Q^{t}$ do

begin

8. $\quad F_{i j}=0$

9. $\quad$ find $N_{i j}$

10. for all $x_{u v}^{t}$ in $N_{i j}$ do

11. $F_{i j, k}=F_{i j, k}+\frac{\left(x_{u v, k}^{t}-\left(\beta_{i j, k}^{t}\right)^{2}\right) \times B_{u v}^{t}}{D\left(x_{i j}^{t}, x_{u v}^{t}\right)}$

end

12. for all q-individuals $q_{i j}^{t}$ in $Q^{t}$ do

begin

13. $v_{i j, k}^{t+1}=\frac{F_{i j, k}}{\eta \times M_{i j}}$

14. $q_{i j, k}^{t+1}=q_{i j, k}^{t}+v_{i j, k}^{t+1}$

end

end

end

The description of the proposed algorithm is as follows: Step 1. This paper uses a cellular structure for population. In the initialization step, the quantum-individuals $q_{i j}^{0}$ are located in a lattice-like population. Then $\left[\alpha_{i j, k}^{0} \beta_{i j, k}^{0}\right]^{T}$ of all $q_{i j}^{0}$ are initialized with $1 / \sqrt{2}$, where $i, j=1,2, S$ is the location of the q-individuals in the lattice, $k=1,2, \ldots, m$, and $m$ is the number of qubits in the individuals. This implies that each qubit individual $q_{i j}^{0}$ represents the linear superposition of all possible states with equal probability. 
Step 3. This step makes a set of binary instants $X^{t}=\left\{x_{i j}^{t} \mid i, j=1,2, \ldots, S\right\}$ at generation $t$ by observing $Q^{t-1}=\left\{x_{i j}^{t-1} \mid i, j=1,2, \ldots, S\right\}$ states, where $X^{t}$ at generation $t$ is a random instant of qubit population and $S$ is the size of lattice. Each binary instant, $x_{i j}^{t}$ of length $m$, is formed by selecting each bit using the probability of qubit, either $\left|\alpha_{i j, k}^{t-1}\right|^{2}$ or $\left|\beta_{i j, k}^{t-1}\right|^{2}$ of $q_{i j}^{t-1}$. Observing the binary bit $x_{i j, k}^{t}$ from qubit $\left[\begin{array}{ll}\alpha_{i j, k}^{t} & \beta_{i j, k}^{t}\end{array}\right]^{T}$ performs as:

$$
x_{i j, k}^{t}= \begin{cases}0 & \text { if } R(0,1)<\left|\alpha_{i j, k}^{t}\right|^{2} \\ 1 & \text { otherwise }\end{cases}
$$

Where $R(.,$.$) is a uniform random number generator.$

Step 4. Each binary instant $x_{i j}^{t}$ is evaluated to give some measure of its objective. In this step, the fitness of all binary solutions of $X^{0}$ are evaluated and stored in $B^{t}$.

Step 5. Next the normalization is performed on $B^{t}$. The normalization is performed as:

$$
B_{i j}=\frac{B_{i j}-\operatorname{Min}}{\operatorname{Max}-\operatorname{Min}}
$$

Where: $\operatorname{Min}^{\prime} \operatorname{minimum}_{i, j=1}^{S}\left(B_{i j}^{t}\right), \quad \operatorname{Max}_{=} \operatorname{maximum}_{i, j=1}^{S}\left(B_{i j}^{t}\right)$

The magnetic field of each particle is normalized in the range of [0-1]. This is because the fitness values of possible solutions are problem dependent. The range of the fitness of the possible solutions can be in any range, since the amount of the magnetic field controls the movement of the particles, we normalize the amount of magnetic field.

Step 6. In this step the mass of all particles is calculated and stored in $M^{t}$ :

$$
M_{i j}^{t}=1+B_{i j}^{t}
$$

Step 7. In this step in the "for" loop, the resultant force of all forces on each particle is calculated.

Step 8. At first the resultant force $F_{i j}$ to particle $x_{i j}^{t}$ is initialized to zero.

Step 9. In the lattice-like structure of QEA population, each particle interacts only with its neighbors i.e. each particle applies its force only to its neighbors. In this step the neighbors of $x_{i j}^{t}$ are considered. The set of neighbors for particle $x_{i j}$ can be defined as $N_{i j}=\left\{x_{i^{\prime} j}, x_{i j^{\prime}}, x_{i{ }^{\prime} j}, x_{i j^{\prime}}\right\}$ Where:

$$
i^{\prime}=\left\{\begin{array}{rr}
i-1 & i \neq 1 \\
S \quad i=1
\end{array} \quad, j^{\prime}=\left\{\begin{array}{l}
j-1 j \neq 1 \\
S \quad j=1
\end{array}, i "=\left\{\begin{array}{rr}
i+1 & i \neq S \\
1 & i=S
\end{array}, j^{\prime \prime}=\left\{\begin{array}{l}
j+1 j \neq S \\
1 \quad j=S
\end{array}\right.\right.\right.\right.
$$

Step 10. In this step, the applied force to particle $x_{i j}^{t}$ by its neighbor's $x_{u v}^{t} \forall x_{u v}^{t} \in$ $N_{i j}$ is calculated.

Step 11. The force which is applied by $x_{u v}^{t}$ to $x_{i j}^{t}$ relates to the distance between two particles and the magnetic field of $x_{u v}^{t}$ and is calculated as:

$$
F_{i j, k}=\frac{\left(x_{u v, k}^{t}-\left(\beta_{i j, k}^{t}\right)^{2}\right) \times B_{u v}^{t}}{D\left(x_{i j}^{t}, x_{u v}^{t}\right)}
$$


Here $F_{i j}$ shows the force applied to q-individual $q_{i j}^{t}$. The part " $x_{u v, k}^{t}-\left(\beta_{i j, k}^{t}\right)^{2}$ ", shows the direction which the q-individual moves and $\left(\beta_{i j, k}^{t}\right)^{2}$ is the probability of $q_{i j}^{t}$ representing state " 1 ". Where $D(.,$.$) is the distance between each pair of$ neighboring particles and is calculated as:

$$
D\left(x_{i j}^{t}\right)=\frac{1}{m} \sum_{k=1}^{m}\left|x_{i j, k}^{t}-x_{u v, k}^{t}\right|
$$

Where $x_{i j}^{t}$ and $x_{u v}^{t}$ are $(i, j)-t h$ and $(u, v)$ - th binary solutions of the population in iteration $t$ respectively and $x_{i j, k}^{t}$ is the $k-t h$ dimension of $(i, j)-t h$ binary solution in iteration $t$. This step is the main step in the proposed algorithm.

Steps $12,13,14$. In these steps the location of q-individuals are updated. Here $\eta$ is the movement coefficient which controls the speed of movement.

The proposed update operator has two advantages. First according to 8 the observed binary solutions with higher fitness have bigger magnetic field $B$ and apply more force to the q-individual, therefore the better binary solutions have more attraction force. Here unlike Q-Gate the movement of q-individuals is not constant throughout the search process and varies for various q-individuals and even various dimensions. Second in the proposed update operator even the inferior binary solutions have effect on the q-individuals but with smaller amplitude. Accordingly the interaction among possible solutions is much more than Q-Gate and the inferior binary solutions participate in the search process. It helps the algorithm escaping from local optima and if the inferior binary solutions are near an optimum, helps the q-individuals to find the optimum.

\subsection{Parameter tuning}

As it is seen in step 13 of MQEA, the proposed algorithm has a parameter of $\eta$. This section tries to find the best parameter for the proposed update operator for several benchmark functions. The size of population for all the experiments is set to 25 , and the parameter is set to $\eta=(1,2,3,4,5,10,15,20,25,30,35,40,45,50)$. Figure 1 shows the parameter setting for the proposed algorithm on Knapsack problem and Generalized Schwefel's Function 2.26. The results are averaged over 30 runs. According to Figure 1, the best parameter for Knapsack problem repair type 1, the best parameter is $\eta=5$, the best parameter for Knapsack penalty type 2 is $\eta=20$ and the best parameter for Generalized Schwefel is 10. This paper finds the best parameter for the proposed update operator for several benchmark functions and the results are summarized in Table 2. As it is clear in Table 2, for all the numerical function problems the best parameter for the proposed update operator is 10 . 
Table 2 Best parameter for the proposed Update operator. The results are averaged over 30 runs

\begin{tabular}{llllllll}
\hline Problem & $\eta$ & Problem & $\eta$ & Problem & $\eta$ & Problem & $\eta$ \\
\hline Kpck Rep 1 & 5 & Kpck Rep 2 & 35 & Kpck Pen 1 & 5 & Kpck Pen 2 & 20 \\
Trap & 2 & Schwefel [16] & 10 & Rastrigin [16] & 10 & Ackley [16] & 10 \\
Griawank [16] & 10 & Penalized 1 [16] & 10 & Penalized 2 [16] & 10 & Kennedy [15] & 10 \\
Michalewicz [15] & 10 & Goldberg [15] & 10 & Sphere [16] & 10 & Rosenbrock [15] & 10 \\
Schwefel 2.21 [16] & 10 & Dejong [15] & 10 & Schwefel 2.22 [16] 10 & & \\
\hline
\end{tabular}

\section{Experimental Results}

The proposed algorithm is compared with the original version of QEA to show the improvement on QEA. The best parameters as found in previous sections are used in order to provide fair comparison between the proposed algorithm and the original version of QEA. The parameters of QEA is set to the best parameters found in [1]. The experimental results are performed on Knapsack problem Penalty type 1 and 2, Knapsack problem Repair types 1 and 2 (see Appendix), Trap problem and fourteen numerical function optimization problems, for the dimension of 100 and 250. The population size of all algorithms for all of the experiments is set to 25; termination condition is set for a maximum of 2000 iterations for Knapsack and Trap problems and 5000 iterations for the fourteen numerical functions. The parameter of QEA is set to Table 1 . The parameter of the proposed update operator is set to the values found in previous section. Due to statistical nature of the optimization algorithms, all results are averaged over 30 runs.

Table 3 shows the experimental results on the proposed magnetic optimization update operator and Q-Gate update operator. According to Table 3, the proposed update operator improves the performance of QEA significantly, and in all the experimental results, the proposed algorithm reaches better results. Additionally, the standard deviation of the best reached results over 30 runs in the proposed update operator is much smaller than Q-Gate. The small STD shows better performance for the proposed algorithm.
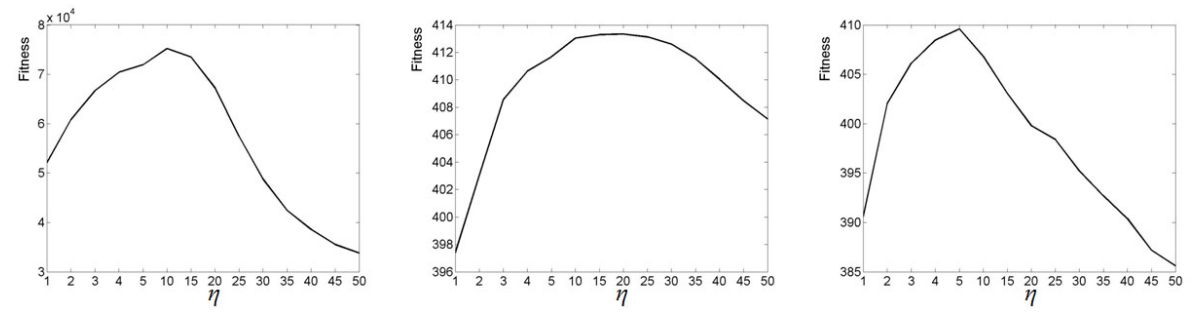

Fig. 1 The effect of the parameter $\eta$ on the performance of the proposed algorithm on Generalized Schwefel's Function 2.26, Knapsack problem Penalty Type 2 and Trap problem. 
Table 3 Experimental results on Knapsack problem, Trap Problem and fourteen numerical function optimization problems. The number of runs is 30. Mean and STD represent the mean of best answers and standard deviation of best answers for 30 runs respectively. $m$ is the dimension of problem. The bold results are the best ones.

\begin{tabular}{|c|c|c|c|c|c|c|c|c|}
\hline \multirow[b]{3}{*}{ Problem } & \multicolumn{4}{|c|}{$m=100$} & \multicolumn{4}{|c|}{$m=250$} \\
\hline & \multicolumn{2}{|c|}{ Q-Gate } & \multicolumn{2}{|c|}{ Magnetic } & \multicolumn{2}{|c|}{ Q-Gate } & \multicolumn{2}{|c|}{ Magnetic } \\
\hline & Mean & STD & Mean & STD & Mean & STD & Mean & STD \\
\hline Kpck Rep 1 & 373.73 & 4.79 & 387.04 & 0.90 & 907.53 & 18.37 & 1008.50 & 3.11 \\
\hline Kpck Rep 2 & 438.44 & 6.77 & 456.32 & 0.02 & 887.43 & 16.27 & 991.73 & 1.46 \\
\hline Kpck Pen 1 & 373.73 & 4.79 & 387.04 & 0.90 & 966.76 & 20.96 & 1086.27 & 4.15 \\
\hline Kpck Pen 2 & 434.97 & 7.20 & 456.29 & 0.05 & 930.56 & 18.26 & 1046.00 & 0.59 \\
\hline Trap & 71.50 & 4.65 & 80.33 & 1.18 & 156.67 & 8.15 & 196.90 & 3.13 \\
\hline Schwefel & $4.2 \times 10^{4}$ & 5558 & $7.6 \times 10^{4}$ & 1304 & $7.9 \times 10^{4}$ & 14101 & $1.8 \times 10^{5}$ & 2207 \\
\hline Rastrigin & -1677 & 259 & -252 & 35 & -5858.41 & 720.78 & -848.38 & 75.77 \\
\hline Ackley & -17.91 & 0.12 & -5.72 & 1.75 & -18.21 & 0.13 & -11.86 & 0.81 \\
\hline Griewank & -32.18 & 5.79 & -0.07 & 0.16 & -127.65 & 23.17 & -1.67 & 0.40 \\
\hline Penalized 1 & $-1.3 \times 10^{5}$ & $2.4 \times 10^{4}$ & -33.45 & 288.06 & $-5.1 \times 10^{5}$ & $1.0 \times 10^{5}$ & -1864 & 1179 \\
\hline Penalized 2 & $-3.0 \times 10^{4}$ & 7014 & -107.60 & 69.05 & $-1.1 \times 10^{5}$ & $2.4 \times 10^{4}$ & -1415 & 566 \\
\hline Michalewicz & 32.99 & 6.33 & 80.97 & 1.372 & 51.79 & 15.07 & 190.24 & 2.86 \\
\hline Goldberg & 46.40 & 3.76 & 89.01 & 1.07 & 93.77 & 12.14 & 212.65 & 2.04 \\
\hline Sphere Model & $-3.3 \times 10^{5}$ & $8.1 \times 10^{4}$ & -1435 & 1176 & $-1.4 \times 10^{6}$ & $2.2 \times 10^{5}$ & $-1.8 \times 10^{4}$ & 4631 \\
\hline Schwefel 2.22 & -4.10 & 0.61 & -0.05 & 0.01 & -5.73 & 0.54 & -0.19 & 0.02 \\
\hline Schwefel 2.21 & -172.70 & 6.15 & -124.64 & 11.57 & -189.44 & 2.14 & -161.94 & 4.91 \\
\hline Dejong & $-2.2 \times 10^{7}$ & $8.3 \times 10^{6}$ & -92603 & 48854 & $-2.6 \times 10^{8}$ & $7.3 \times 10^{7}$ & $-2.2 \times 10^{6}$ & $7.7 \times 10^{5}$ \\
\hline Rosenbrock & $-7.7 \times 10^{4}$ & $2.0 \times 10^{4}$ & -2460 & 1311 & $-3.6 \times 10^{5}$ & $9.9 \times 10^{4}$ & $-1.2 \times 10^{4}$ & 3488 \\
\hline Kennedy & -1.26 & 0.83 & -0.0003 & 0.0003 & -18.17 & 7.61 & -0.003 & 0.002 \\
\hline
\end{tabular}

\section{Conclusion}

This paper proposes a novel alternative for Q-Gate update operator. In the proposed update operator the q-individuals are some magnetic particles being attracted to binary solutions based on their fitness. In comparison with Q-Gate the proposed update operator has two advantages. First the movement of q-individuals is not constant throughout the search process and varies for various q-individuals and even various dimensions. Second in the proposed update operator even the inferior binary solutions have effect on the q-individuals but with smaller amplitude. Accordingly the interaction among possible solutions is much more than Q-Gate and the inferior binary solutions participate in the search process. The proposed algorithm has a parameter; this paper has also investigated the effect of the parameter for several benchmark functions. The proposed update operator is tested on several benchmark functions, and experimental results shows better performance for the proposed operator than Q-Gate. In our future works we will focus on some operators to improve the performance of the proposed algorithm and apply the proposed algorithm on some real problems. 


\section{References}

1. K. Han and J. Kim, Quantum-inspired evolutionary algorithm for a class of combinatorial optimization, IEEE Transactions on. Evolutionary Computing, Vol. 6. No 6, 2002.

2. Y. Li, Y. Zhang, R. Zhao and L. Jiao, The immune quantum-inspired evolutionary algorithm, IEEE International Conference on Systems, Man and Cybernetics, 2004.

3. Y. Wang, X. Feng, Y. Huang, W. Zhou, Y. Liang and C. Zhou, A Novel Quantum Swarm Evolutionary Algorithm for Solving 0-1 Knapsack Problem, Springer, Advances in Natural Computation, Vol 3611. pp 698-704.

4. H.Gao, G. Xu and Z. Wang, A Novel Quantum Evolutionary Algorithm and Its Application, The Sixth IEEE World Congress on Intelligent Control and Automation, 2006.

5. Y. Yu, Y. Tian and Z. Yin Hybrid Quantum Evolutionary Algorithms Based on Particle Swarm Theory, 1ST IEEE Conference on Industrial Electronics and Applications, 2006.

6. X. You, S. Liu and D. Shuai On Parallel Immune Quantum Evolutionary Algorithm Based on Learning Mechanism and Its Convergence, Springer, Advances in Natural Computation, 903-912. 2006.

7. D. Cruz, A.V.A. Vellasco, M.M.B. Pacheco, Quantum-Inspired Evolutionary Algorithm for Numerical Optimization, IEEE Congress on Evolutionary Computation, 2006.

8. X.Y. Feng, Y. Wang, H.W. Ge, C.G. Zhou and Y.C. Liang, Quantum-Inspired Evolutionary Algorithm for Travelling Salesman Problem, Springer, Computational Methods, 1363-1367, pp 2007.

9. M.D. Platelt,S. Schliebs, N. Kasabov, , A versatile quantum-inspired evolutionary algorithm, IEEE Congress on Evolutionary Computation, 2007.

10. S.R. Hutsell and G.W. GreenwoodApplying evolutionary techniques to quantum computing problems, IEEE Congress on Evolutionary Computation, 2007.

11. M.-H. Tayarani- N. and M.- R. Akbarzadeh-T A Cellular Structure and Diversity Preserving operator in Quantum Evolutionary Algorithms, IEEE World Conference on Computational Intelligence 2008.

12. M.-H. Tayarani- N. and M.- R. Akbarzadeh-T A Sinusoid Size Ring Structure Quantum Evolutionary Algorithm, IEEE International Conference on Cybernetics and Intelligent Systems Robotics, Automation and Mechanics 2008.

13. M.-H. Tayarani- N. And M.-R. Akbarzadeh-T Probabilistic Optimization Algorithms for Numerical Function Optimization Problems, IEEE International Conference on Cybernetics and Intelligent Systems Robotics, Automation and Mechanics 2008.

14. M.-H. Tayarani- N and M.- R. Akbarzadeh-T Magnetic Optimization Algorithm, A New Synthesis, IEEE World Conference on Computational Intelligence 2008.

15. A.-R. Khorsand, M.-R. Akbarzadeh-T Quantum Gate Optimization in a Meta-Level Genetic Quantum Algorithm, IEEE International Conference on Systems, Man and Cybernetics, 2005.

16. W. Zhong, J. Liu, M. Xue, L. Jiao A Multi-agent Genetic Algorithm for Global Numerical Optimization, IEEE Trans. Sys, Man and Cyber. vol. 34, pp. 1128-1141, 2004.

17. V. K. Koumousis, C. P. Katsaras A Saw-Tooth Genetic Algorithm Combining the Effects of Variable Population Size and Reinitialization to Enhance Performance, IEEE Trans. Evol. Comput. vol. 10, pp. 19-28, 2006. 